\section{Follow-Up for Gastric Cancer: How Extensive and Intensive Should It Be?}

\section{TO THE EDITORS:}

Usually after complete resection (R0), patients with gastric cancer undergo a follow-up including endoscopy, computer tomography (CT), and serum iron measures. It is unknown whether this follow-up strategy or a more extensive strategy associated with increased costs, including for example positron emission tomography (PET), is beneficial.

Even after overcoming the current economic crisis, payers and regulators will need data for the efficiency and usefulness of follow-up strategies in order to continue to pay. For example, there is currently controversy on public health care reform in the United States. We urgently need to reduce the cost of care, notes Bodenheimer and colleagues. ${ }^{1}$

In the absence of evidence-based recommendations for follow-up and the very poor prognosis of patients developing recurrence after standardized D2 gastrectomy, intensive and high-cost imaging evaluation is questionable.

On this controversial topic, Hur and colleagues provide useful information based on nationwide survey in Korea, where gastric cancer is epidemic. ${ }^{2}$ The authors sent out questionnaires to 205 members of the Korean Gastric Cancer Association (KGCA) about follow-up schedules and methodologies. Follow-up studies were carried out more often in patients with advanced gastric cancer than with early gastric cancer in the first follow-up year (every 3 months vs every 6 months, respectively) and thereafter. After surgery, most responder's members of the KGCA used CT for imaging, carcinoembryonic antigen (CEA) and carbohydrate antigen (CA) 19-9 as tumor markers, and serum iron among follow-up measures. The authors conclude that clinicians use a variety of approaches in Korea regarding the extent of follow-up and methodologies after curative resection for gastric cancer. They propose a multicenter randomized trial to compare routine follow-up with intensive schedules.

Current, phase III randomized trial based guidelines recommend standardized surgery and adjuvant treatment. In Japan, gastrectomy with D2 lymphadenectomy is followed by postoperative adjuvant chemotherapy for stages II and III disease. In the United States and Europe the extent of lymphadenectomy varies, but postoperative chemoradiotherapy or perioperative chemotherapy, respectively, are the standards of care. After such an aggressive multimodal treatment, the evidence of recurrence is nearly always fatal. Therefore, intensive and extensive postoperative screening to identify recurrence at an early, potentially curable stage is in most cases unrealistic.

Despite this disappointing outcome of patients with evident recurrence, prospective cost-effectiveness studies are required. However, all efforts should be focused to prevent relapse. At the advanced stage, the accumulation of a tremendous number of driver mutations in multiple genes likely makes recurrence an incurable disease even for the future.

C. Bali, MD, D. H. Roukos, MD, and D. E. Ziogas, MD Department of Surgery, School of Medicine, University of Ioannina, Ioannina, Greece

e-mail: deziogas@hotmail.com

Published Online: 4 December 2009

(C) Society of Surgical Oncology 2009

\section{REFERENCES}

1. Bodenheimer T, Grumbach K, Berenson RA. A lifeline for primary care. N Engl J Med. 2009;360:2693-6.

2. Hur H, Song KY, Park CH, Jeon HM. Follow-up strategy after curative resection of gastric cancer: a nationwide survey in Korea. Ann Surg Oncol. 2009 Sep 24; doi:10.1245/s10434-009-0676-1 (Epub ahead of print). 